\title{
Relaxation Dynamics Near Ferroelectric Phase Transitions and the Central-Peak Phenomenon
}

\author{
V.B. Kokshenev \\ Departamento de Física, ICEx, Universidade Federal de Minas Gerais, \\ Caixa Postal 702, CEP 30123-970, Belo Horizonte, MG, Brazil \\ electronic address: valery@fisica.ufmg.br
}

Revised form received on 12 October, 2000

\begin{abstract}
The instability of the paraelectric phase in ferroelectrics, driven by thermal fluctuations, is discussed on the basis of the quantum three-dimensional spin- $1 / 2$ transverse-field Ising model (TIM) within the framework of the Green function method. The two-step critical dynamics of the TIM is analyzed through the ferroelectric order-parameter fluctuation spectra observed above the critical temperature $T_{c}$. The spectra profiles are given near $T_{c}$ in explicit form. The slow-down exponents $\Delta_{c}=\frac{5}{4}$ and $\Delta_{s}=\frac{1}{4}$ are deduced for the slow and fast parts of structural relaxation and are compared with those known from the literature.
\end{abstract}

\section{Introduction}

After Elliot [1] and Stinchcombe [2] it has been repeatedly recognized that the pseudo-spin Transverse Ising Model (TIM) [3],

$$
H=-\Gamma \sum_{f}^{N} S_{x f}-\frac{1}{2} \sum_{f f^{\prime}} J_{f f^{\prime}} S_{z f} S_{z f^{\prime}},
$$

is generic to describe the critical dynamics observed in ferroelectrics and antiferroelectrics near structural phase transitions that occur at a critical temperature $T_{c}$. The transverse field $\Gamma$ provides specific dynamics near $T_{c}$. This is due to short-range thermal fluctuations of the local order parameter that can be treated in terms of compact correlation regions (or clusters) of the local polarization. Critical dynamical effects are directly observed in the ferroelectric order-parameterfluctuation spectra both below and above $T_{c}$ and are known as the central peak $(\mathrm{CP})$ phenomenon.

The main qualitative features of the $\mathrm{CP}$ are as follows. The diffusive-type central-mode $\left(\operatorname{Re} \omega_{c}=0\right.$, $\left.\operatorname{Im} \omega_{c} \neq 0\right)$ appears in the dynamical spectra, in addition to the renormalized soft mode $\omega_{s}$ related to the side-band peaks. Near $T_{c}$ the central-mode intensity $I(\omega, T)$ diverges but the soft mode remains finite $\left(\operatorname{Im} \omega_{s} \neq 0\right)$. The CP phenomenon appears to be associated with all structural phase transitions [4], and has been intensively studied by a great variety of experimental techniques, namely, by neutron, light and Mössbauer scattering, electron and nuclear paramagnetic resonances, dielectric dispersion and ultrasound [5]. In this work we give a microscopic description of the observed CP phenomena based on the critical dynamics of the 3-dimensional (3D) TIM (1) developed through the Green function method.

\section{CP Microscopical Descrip- tion}

We discuss the CP phenomenon in terms of the wellknown phenomenological description proposed by several authors [5]. This description introduces the auxiliary parameters $\delta$ (coupling strength) and $\frac{1}{\nu}$ (Debye relaxation time) through the dynamical spectral profiles,

$$
I(\omega, T) \propto \frac{1}{\omega} \operatorname{Im} G_{0}(\omega, T),
$$

and

$$
G_{0}(\omega, T) \propto\left[\omega_{0}^{2}-\omega^{2}-i \omega \frac{\delta^{2}}{\nu-i \omega}\right]^{-1} .
$$

The auxiliary parameters $\delta$ and $\nu$ of unknown nature were suggested to describe the interaction of a trial undamped soft mode $\omega_{0}$ with some unspecified degree of freedom, called a relaxation mode. The auxiliary parameters can in turn be derived from the observed spectra and given in terms of the frequencies $\omega_{s}$ and $\omega_{c}$ and their widths, respectively, $\gamma_{s}$ and $\gamma_{c}$. Thus, as $T \rightarrow T_{c}$, the $\mathrm{CP}$ behavior is given by

$$
\omega_{s}^{2} \rightarrow \omega_{\infty}^{2}=\omega_{0}^{2}+\delta^{2}, \gamma_{c} \rightarrow \nu \frac{\omega_{0}^{2}}{\omega_{\infty}^{2}} .
$$

From the Heisenberg equations of the spin-1/2 TIM (1) one obtains the Green function 


$$
G_{f f^{\prime}}(t)=-i \Theta(t)<\left[S_{z f}(t) S_{z f^{\prime}}(0)\right]>_{T}
$$

with $<S_{y f}(t)>_{T}=<S_{z f}(t)>_{T}=0, \quad$ and $<S_{x f}(t)>_{T}=\sigma(T)=\frac{1}{2} \tanh \left(\frac{\Gamma}{2 T}\right)$. The Fourier trans- form $G_{q}(\omega, T)$ has been developed up to the forth order and consistently reduced by application of the symmetrized Tyablikov decoupling scheme. The closed chain-equation system was found [6] in explicit form,

$$
G_{q}(\omega, T)=\frac{\Gamma \sigma(T)}{\pi}\left[\omega^{2}-\omega_{q}^{2}(T)-\Delta_{q}(\omega, T)\right]^{-1} \quad \text { with } \quad \omega_{q}^{2}(T)=\Gamma\left[\Gamma-J_{q} \sigma(T)\right],
$$

where $J_{q}$ stands for the Fourier-site 3D transformed nearest-neighbor exchange energy and the polarization "operator" is

$$
\Delta_{q}(\omega, T)=\frac{\omega^{2}<S_{z}^{2}>_{T}}{N} \sum_{q^{\prime}} \frac{J_{q^{\prime}}^{2}}{\omega^{2}-\omega_{q^{\prime}}^{2}(T)}-\frac{\Gamma^{2}<S_{x}^{2}>_{T}^{2}}{N} J_{q} \sum_{q^{\prime}} \frac{J_{q^{\prime}}}{\omega^{2}-\omega_{q^{\prime}}^{2}(T)} .
$$

For $q \rightarrow 0$ and $T \rightarrow T_{c}^{+}$, Eqs. (5) and (6) provide the following CP phenomenological auxiliary parameters,

$$
\omega_{0} \rightarrow \Gamma\left(\frac{T}{T_{c}}-1\right)^{1 / 2}, \delta \rightarrow \Gamma \quad \text { and } \quad \nu \rightarrow \Gamma\left(\frac{T}{T_{c}}-1\right)^{1 / 4} .
$$

as well the observable parameters,

$$
\omega_{s} \rightarrow \Gamma, \gamma_{s} \rightarrow \Gamma\left(\frac{T}{T_{c}}-1\right)^{1 / 4} \text { and } \gamma_{c} \rightarrow \Gamma\left(\frac{T}{T_{c}}-1\right)^{5 / 4} .
$$

The critical regime is defined by the conditions $\delta>>$ $\nu>>\omega_{0}$. Distinct microscopic approaches to the $\mathrm{CP}$ problem, based on the TIM (1) suggest a different description of auxiliary parameters $\delta$ and $\nu$ (for discussion see Refs. 5,7).

The analysis of the CP phenomenon is given in the TIM rigid-lattice approximation but there is the problem of the stability of the solutions (8) against lattice vibrations characteristic of real crystals. The stability of the thermal-fluctuation mechanism was investigated [8] by a complete qualitative analyses, including lattice distortions, spin-phonon interactions and phonon unharmonicity, carefully accounted through the ETIM, an extended version of the zero-order lattice-vibration TIM. The ETIM was introduced in the second-order lattice-vibration approximation, that, in a way, generalizes the well known (see e.g. Ref.3) first-order latticevibration Kobayashi model. The ETIM is proposed [8] as a credible model for real crystals that expose structural order-disorder transition.

\section{Summary}

We have discussed the relaxation phenomenon observed near the structural transition in three-dimensional hydrogen-bonded ferroelectrics above the transition temperature $T_{c}$. The analysis is given through the instability of paraelectric phase studied within the scope of the 3D spin-1/2 TIM by the Green-function method. The observed central-mode intensity $(2,3)$ is described microscopically near $T_{c}$ by Eqs. (7) and (8). The mean-field dynamic description, given by the trial soft mode and $\omega_{0}(T)$, with $\operatorname{Re} \omega_{0} \propto\left(T-T_{c}\right)^{1 / 2}$ and $\operatorname{Im} \omega_{0}$ $=0$, is explained by accounting for the short-range order-parameter correlations and is given in terms of the divergent relaxation scale $\tau(T)$. The latter, in turn, is given by the diffusive-type, central-mode $\omega_{c}(T)$ $\left(\operatorname{Re} \omega_{c}=0\right.$ and $\left.\tau_{c}=\operatorname{Im} \omega_{c}^{-1} \propto\left(T-T_{c}\right)^{-\Delta_{c}}\right)$ and the overdamped soft-mode $\omega_{s}(T)\left(\operatorname{Re} \omega_{s} \sim \Gamma\right.$ and $\tau_{s}=$ $\left.\operatorname{Im} \omega_{s}^{-1} \propto\left(T-T_{c}\right)^{-\Delta_{s}}\right)$ order-parameter excitations. As follows from Eq.(8) the slow-down exponents are, respectively, $\Delta_{c}=\frac{5}{4}$ and $\Delta_{s}=\frac{1}{4}$. One of these results can be compared with the numerical estimate $\Delta_{c}=1.26$ elaborated in Ref.9.

The instability of the ferroelectric phase below $T_{c}$ was additionally analyzed on the basis of the classical Ising model by the Thompson method [10,11]. The following slow-down exponents, namely, $\Delta_{c}^{\prime}=\frac{5}{4}$ and $\Delta_{s}^{\prime}=\frac{5}{16}$, were deduced in the case of the real 3D space. We see that the Ising-model critical dynamics, associated with $T_{c}$-critical dynamics in hydrogen-bonded ferroelectrics [1-5], is driven by thermal order-parameter fluctuations (intrinsic CP mechanism) and does not depend on the quantum model correlations characteristic of the TIM.

Thanks are due to Alaor S. Chaves, Francisco César 
Sá Barreto, and Paulo Roberto da Silva, for numerous helpful discussions, and to Ronald Dickman for a critical reading of the manuscript. The work was supported by the Brazilian agencies CNPq and FAPEMIG.

\section{References}

[1] R.J. Elliot, in "Structural Phase Transitions and Soft Modes" ( eds. E. J. Samuelson, E. Andersen and J. Feder, Oslo: Universitetsforlaget, 1971), p. 235.

[2] R.B. Stinchcomb, J. Phys. C 6, 2459, 2484, 2507 (1973).

[3] R. Blinc, B. Zeks, "Soft Modes in Ferrorelectrics and Antiferroelectrics" ( ed. Wohlfart E. P., Amsterdam: North-Holland Publishing Company, 1974).

[4] T. Riste, E.J. Samuelson and K. Otnes, Solid State Commun. 9, 1455 (1971).
[5] A.D. Bruce and R.A. Cowley, Adv. Phys. 29, 219 (1980).

[6] V.B. Kokshenev, M.C. Nemes and J.I. Kim, Solid State Commun. 98, 421 (1996).

[7] V.B. Kokshenev and A.S. Chaves, J. Phys.: Condens. Matter 7, 5371(1995).

[8] V.B. Kokshenev and A.S.T. Pires, Phys. Stat. Sol. (b) 197, 333 (1996).

[9] J.M. Wesselinowa and A.T. Apostolov, Solid State Commun. 101, 343 (1997).

[10] V.B. Kokshenev and P.R. Silva, Mod. Phys. Lett., B 12, 265 (1998).

[11] P.R. Silva and V.B. Kokshenev, Braz. Journ. Phys., this issue. 